\title{
Investigation of elastic constants and ultimate strengths of Korean pine from compression and tension tests
}

\author{
Na Yang ${ }^{1} \cdot$ Lei Zhang ${ }^{2}$
}

Received: 27 February 2017 / Accepted: 1 October 2017 / Published online: 18 December 2017

(c) The Japan Wood Research Society 2017

\begin{abstract}
Korean pine (Pinus koraiensis) is a wood species recently adopted in China for the rehabilitation of traditional timber buildings. This paper investigates its mechanical properties with laboratory tests on typical specimens to obtain the moduli of elasticity, Poisson's ratios, shear moduli, coefficients of mutual influence, crushing strengths and tension strengths in various directions. Highly different failure patterns of specimens in compression test were observed when loaded in different directions relative to the grain while only brittle failure mode was observed for tension specimens. The measured parameters of Korean pine were compared with those obtained from theory of orthotropic elasticity, the empirical formula and the Norris failure criterion, good agreements were reached for all examinations in general which indicate it is admissible to treat Korean pine as ideal orthotropic material.
\end{abstract}

Keywords Clear wood $\cdot$ Off-axis loading $\cdot$ Elastic constants $\cdot$ Crushing strengths $\cdot$ Tension strengths

\section{Introduction}

In recent years, the rehabilitation of traditional timber structures which are recognized as valuable culture heritages becomes more and more important in China. Timber once used in the construction of these wooden buildings, such as Machilus sp. and Phoebe sp., is in shortage. This gives rise to the need to find substitutes when the damaged components in these structures are required to be replaced. Korean pine (Pinus koraiensis Sieb. et Zucc.) is one wood species newly adopted for the restoration of important historical structures including the Forbidden City of China. The elastic constants and ultimate strengths in different directions of Korean pine have seldom been reported before. It is, therefore, crucial to carry out experimental studies to have a basic knowledge of its mechanical properties, thus evaluate its suitability as substitute wood species.

Static experimental methods to obtain the mechanical properties of wood could be classified into two categories.

Na Yang

nyang@bjtu.edu.cn

1 School of Civil Engineering, Beijing Jiaotong University, Beijing 100044, China

2 Department of Civil and Environmental Engineering, University of Alberta, Edmonton T6G 1H9, Canada
Classical methods are based on mechanical models in which explicit relationships relating the mechanical properties with specimen geometry, applied load and strain measurements can be proposed by creating homogeneous and simple stress state in the specimens. By simply applying compression [1-4], tension [5, 6], shear [7], bending [8] or torsion [9] loads onto the specimens, the elastic constants and ultimate strengths of wood could be readily obtained from predetermined formulas. In novel methods such as full-field measurement methods [10], however, heterogeneous and complex stress and strain states are actually created in the interest region of the specimens. After the deformation of the specimens are captured by digital cameras, the stiffness parameters of wood can be identified based on numerical computational algorithms such as using virtual field method $[11,12]$. Having different features, these two experimental methods are adopted depending on accuracy of the measured results and simplicity of the test procedure.

In this study, uniaxial compression and tension tests were conducted to characterize the mechanical properties of Korean pine. The loading directions of specimens in the test include those in principal material directions and those in off-axis directions. The elastic constants of Korean pine including moduli of elasticity, shear moduli, Poisson's ratios and coefficients of mutual influence are measured and compared with the theoretical results from theory of elasticity. 
Crushing strengths and tension strengths of Korean pine are also obtained and compared with the Hankinson's formula and orthotropic failure criterion. These examinations are aimed to determine whether it is admissible to treat Korean pine as ideal orthotropic material.

\section{Experiment}

\section{Material}

Korean pine (Pinus koraiensis Sieb. et Zucc.) grown in northeast China with a mean oven-dry density of $0.430 \mathrm{~g} /$ $\mathrm{cm}^{3}$ was used. All specimens for the tests were cut from a same lumber. The sawn and planed boards from the lumber were dried in a kiln for a couple of days before specimens were prepared. All specimens have been carefully inspected to avoid defects such as knots, checks or grain distortions so they can be considered as small clear wood specimens. The specimens were then stored in a chamber of constant temperature environment at $20{ }^{\circ} \mathrm{C}$ and $65 \%$ relative humidity until they achieved a stable and equilibrium state with $12 \pm 1 \%$ moisture content [13].

\section{Specimen preparation}

Specimens for both compression and tension tests were prepared in ten different directions in this study. Figure 1 shows the compression specimens in various directions where legends L, R and T represent longitudinal, radial and tangential directions, respectively. Other than the specimens prepared in principal directions, seven types of off-axis specimens with $15^{\circ}$ interval in LR plane (LR15 $, \mathrm{LR} 30^{\circ}, \mathrm{LR} 45^{\circ}$, $\mathrm{LR}^{\circ} 0^{\circ}$ and $\left.\mathrm{LR} 75^{\circ}\right), 45^{\circ}$ interval in $\mathrm{LT}$ plane $\left(\mathrm{LT} 45^{\circ}\right)$ and $45^{\circ}$ interval in TR plane $\left(\mathrm{TR} 45^{\circ}\right)$ were prepared. The angle

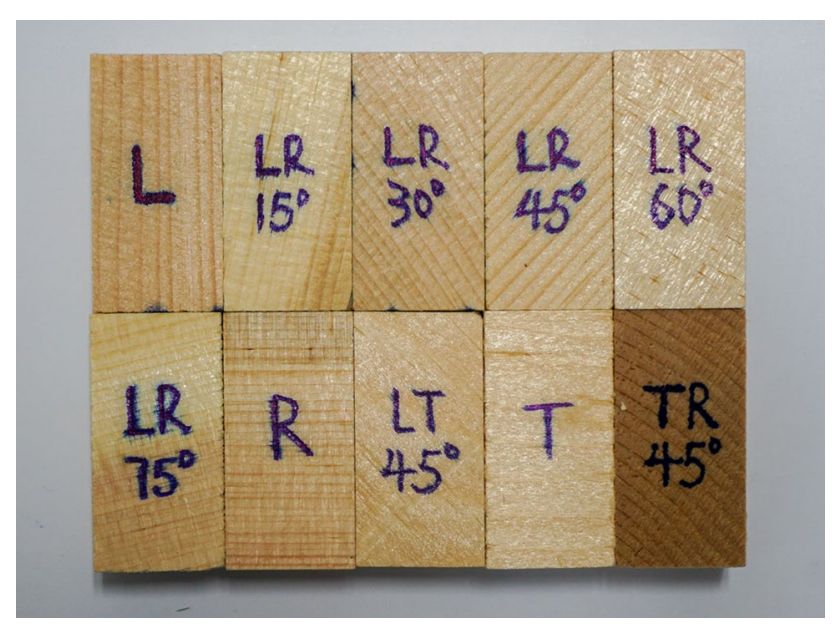

Fig. 1 Compression specimens in different directions in LR or LT planes represents the angle between loading direction and fibers' direction of specimens (grain angle), while the angle in TR plane represents the angle between loading direction and rings' direction of specimens (ring angle). Tension specimens were also prepared with these orientations. Totally ten to thirteen specimens were tested in each direction in the test.

Specimens for compression test are prismatic with a height of $40 \mathrm{~mm}$ and a cross-sectional area of $20 \mathrm{~mm} \times 20 \mathrm{~mm}$ as shown in Fig. 2a, b. The height-to-width ratio of the compression specimens is same as some previous studies [1, 14]. Specimen with this configuration can ensure accurate measurement of elastic parameters of the material and investigation of large deformation of the specimen simultaneously [1]. The stress induced along the loading direction for compression specimens $\sigma_{x}$ can be calculated by

$\sigma_{x}=\frac{F}{A}$

in which $F$ is the load recorded by the data acquisition system and $A$ represents the actual cross-sectional area of specimens measured before test.

The size of tension specimen is shown in Fig. 2c. This specimen is similar to but a little bigger than the specimen adopted by Yoshihara and Ohta [5]. The total length of the specimen is $150 \mathrm{~mm}$ and the length at middle straight part is $50 \mathrm{~mm}$. To avoid stress concentration effect at the specimen ends as well as to protect the specimen from being squashed by the clamping heads of the testing machine, fraxinus mandshurica (Fraxinus chinensis Roxb) lamellas were bonded to both ends of the specimen using polyvinyl acetate emulsion.

When specimens fail under tension load, only those with material rupture at middle straight part will be taken as valid samples. Assuming $t$ and $w$ are the thickness and width of the section at middle straight part, the tensile strength of Korean pine $\sigma_{\mathrm{t}}$ can be calculated as

$\sigma_{\mathrm{t}}=\frac{P_{\max }}{t w}$

where $P_{\max }$ is the maximum load recorded by the load cell.

An INSTRON 5582 universal testing machine with a load capacity of $100 \mathrm{kN}$ was used to apply the load to the specimens at a crosshead speed of $1.0 \mathrm{~mm} / \mathrm{min}$. The load-displacement/strain relations of different specimens were recorded with a TDS-530 data acquisition system.

\section{Calculation of elastic constants}

The constitutive laws characterizing the orthotropic material in its principal directions and off-axis directions are different. For specimens loaded in main directions, the stress-strain relationship can be expressed as 
Fig. 2 Configuration of compression and tension specimens in which $\theta$ represents off-axis angle

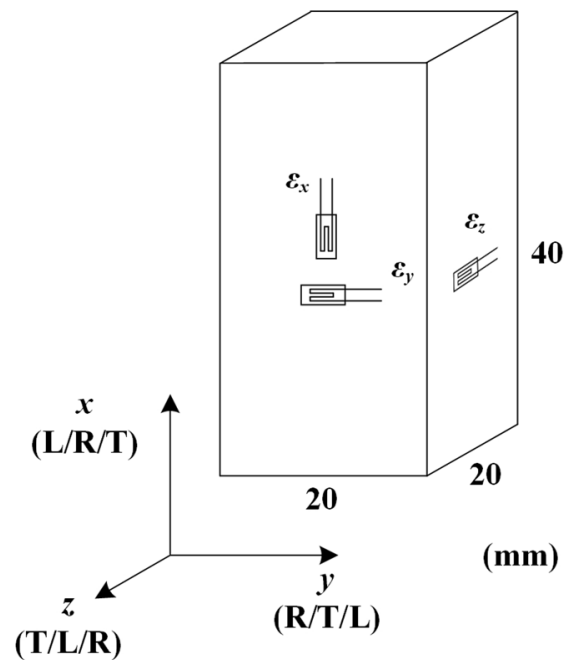

(a) Compression specimen loaded in principal material direction

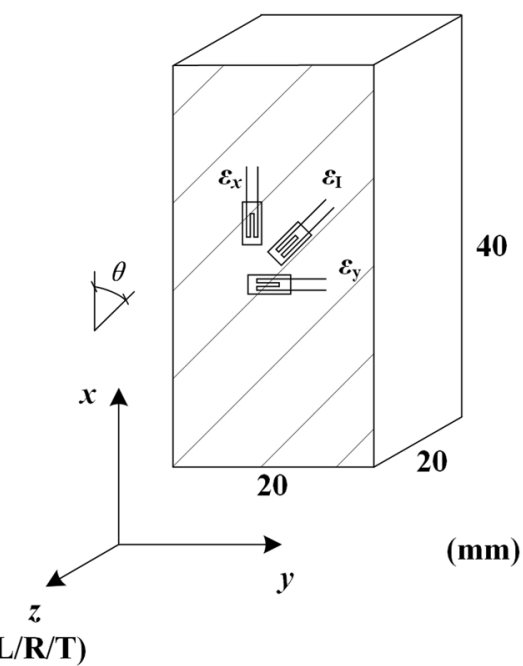

(b) Compression specimen loaded in off-axis material direction

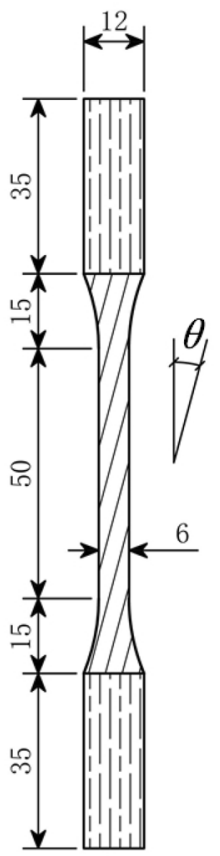

front view

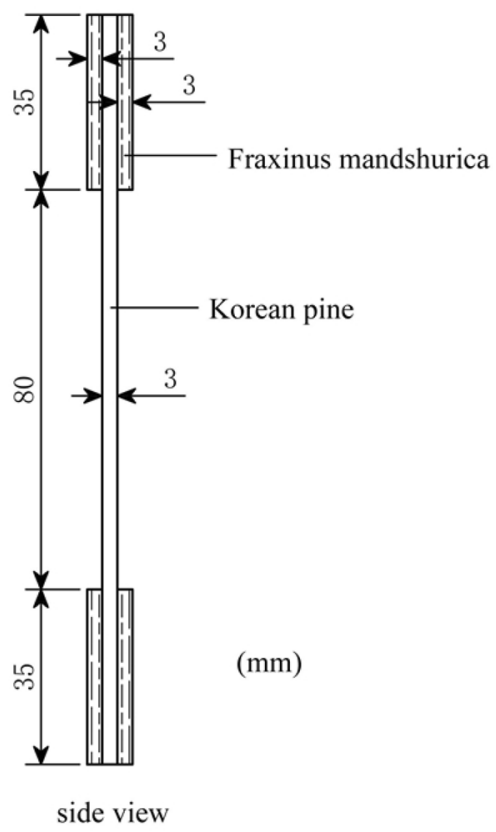

(c) Dimensions of tension specimen

$$
\begin{aligned}
{[\varepsilon] } & =[S][\sigma]=\left[\begin{array}{l}
\varepsilon_{1} \\
\varepsilon_{2} \\
\gamma_{12}
\end{array}\right]=\left[\begin{array}{lll}
S_{11} & S_{12} & 0 \\
S_{21} & S_{22} & 0 \\
0 & 0 & S_{33}
\end{array}\right]\left[\begin{array}{l}
\sigma_{1} \\
\sigma_{2} \\
\tau_{12}
\end{array}\right] \\
& =\left[\begin{array}{ccc}
\frac{1}{E_{1}} & \frac{-v_{21}}{E_{2}} & 0 \\
\frac{v_{12}}{E_{1}} & \frac{1}{E_{2}} & 0 \\
0 & 0 & \frac{1}{G_{12}}
\end{array}\right]\left[\begin{array}{l}
\sigma_{1} \\
\sigma_{2} \\
\tau_{12}
\end{array}\right]
\end{aligned}
$$

where $\varepsilon_{1}$ and $\varepsilon_{2}$ represent normal strains and $\gamma_{12}$ represents shear strain, $\sigma_{1}$ and $\sigma_{2}$ represent normal stresses and $\tau_{12}$ represents shear stress. There are four independent elastic constants in the compliance matrix $[S]$ which are the moduli of elasticity $E_{1}$ and $E_{2}$, major Poisson's ratio $v_{12}$ and shear modulus $G_{12}$. Once these parameters are determined, minor Poisson's ratio $v_{21}$ can be obtained based on the symmetric feature of matrix $[S]$. 
In practical use of wood as construction material, however, external loads are not necessarily coinciding with its principal material directions. This phenomenon is studied with the off-axis specimens in the test with their geometry coordinate systems deviating from the material coordinate system. The stress-strain relationship for an orthotropic body in its off-axis directions is

$$
\begin{aligned}
{[\varepsilon] } & =[\bar{S}][\sigma]=\left[\begin{array}{c}
\varepsilon_{x} \\
\varepsilon_{y} \\
\gamma_{x y}
\end{array}\right]=\left[\begin{array}{lll}
\bar{S}_{11} & \bar{S}_{12} & \bar{S}_{13} \\
\bar{S}_{21} & \bar{S}_{22} & \bar{S}_{23} \\
\bar{S}_{31} & \bar{S}_{32} & \bar{S}_{33}
\end{array}\right]\left[\begin{array}{c}
\sigma_{x} \\
\sigma_{y} \\
\tau_{x y}
\end{array}\right] \\
& =\left[\begin{array}{ccc}
\frac{1}{E_{x}} & \frac{-v_{y x}}{E_{y}} & \frac{\eta_{x, y y}}{G_{x y}} \\
\frac{v_{x y}}{E_{x}} & \frac{1}{E_{y}} & \frac{\eta_{y, x y}}{G_{x y}} \\
\frac{n_{x y, x}}{E_{x}} & \frac{\eta_{x y y y}}{E_{y}} & \frac{1}{G_{x y}}
\end{array}\right]\left[\begin{array}{c}
\sigma_{x} \\
\sigma_{y} \\
\tau_{x y}
\end{array}\right]
\end{aligned}
$$

where stress and strain components have similar meanings with those in Eq. (3) and subscripts $x$ and $y$ denote geometry axes of off-axis specimens as shown in Fig. $2 b$.

There are more non-zero terms in the compliance matrix $[\bar{S}]$ indicating the orthotropic material appears to be anisotropic in off-axis directions. The terms $\eta_{x, x y}, \eta_{y, x y}$ and $\eta_{x y, x}, \eta_{x y, y}$ defined by Jones [15] as the coefficients of mutual influence of the first and second types characterize the coupling effect between shear stress and normal strain, and normal stress and shear strain, respectively (the order of subscripts indicate an effect-cause relationship). Although the compliance matrix $[\bar{S}]$ is more complicated, it can be readily obtained from transformation of $[S]$ with consideration of the off-axis angle.

In this study, the elastic constants of Korean pine were measured from compression test to avoid problematic influence of end constraints that typically accompany tensile test. Uniaxial strain gauges (BE120-3AA with a grid dimension of $2.8 \times 2 \mathrm{~mm}^{2}$ ) bonded at the centers of principal planes of compression specimens are illustrated in Fig. 2a, b. Two sets of identical strain gauges were bonded to two opposite faces of each specimen and the measured results were averaged to eliminate possible unsymmetrical loading effect.

The moduli of elasticity (MOE) in different directions $E_{x}$ can be regressed as the slope of the initial straight segment of compressive stress-strain curves expressed as

$E_{x}=\Delta \sigma_{x} / \Delta \varepsilon_{x}$

The Poisson's ratios can be calculated as the ratio of strain in lateral direction $\varepsilon_{y}$ to the strain in vertical direction $\varepsilon_{x}$ and takes the opposite value as shown in the following equation:

$v_{x y}=-\Delta \varepsilon_{y} / \Delta \varepsilon_{x}$

Poisson's ratios were only measured in principal planes in this study. Based on the way the strain gauges bonded at the specimens, two Poisson's ratios can be measured from specimens in principal directions (Fig. 2a) while only one Poisson's ratio can be measured from off-axis specimens (Fig. 2b).

For specimens loaded in off-axis directions, normal stress $\sigma_{x}$ can induce shear strain $\gamma_{x y}$ which can be calculated from

$\gamma_{x y}=\left(\varepsilon_{1}-\varepsilon_{x} \cos ^{2} \theta-\varepsilon_{y} \sin ^{2} \theta\right) / \sin \theta \cos \theta$

where $\theta$ represents the off-axis angle (could be grain angle or ring angle depending on the loading direction).

The second type of coefficient of mutual influence $\eta_{x y, x}$ represents the coupling effect between compressive stress $\sigma_{x}$ and induced shear strain $\gamma_{x y}$. This non-dimensional parameter is defined as the ratio of strains in the following equation:

$\eta_{x y, x}=\frac{\gamma_{x y}}{\varepsilon_{x}}$

The first type of coefficient of mutual influence cannot be obtained from this test, thus is not discussed.

The shear moduli $G_{\mathrm{LR}}, G_{\mathrm{LT}}$ and $G_{\mathrm{RT}}$ in three principal planes were measured from their corresponding $45^{\circ}$ off-axis specimens and calculated from the following equation:

$G_{i j}=\Delta \sigma_{x} /\left[2 \Delta\left(\varepsilon_{x}-\varepsilon_{y}\right)\right], \quad i, j=\mathrm{L}, \mathrm{R}, \mathrm{T}$

According to the theory of orthotropic elasticity and considering tensor transformation law, the MOE $E_{\theta}$, Poisson's ratios $v_{\theta}$ and coefficients and mutual influence $\eta_{x y, x}^{\theta}$ in arbitrary directions of an orthotropic body can be obtained based on the knowledge of elastic parameters in principal directions as shown in Eq. (10)-(12):

$E_{\theta}=\left[\frac{\cos ^{4} \theta}{E_{1}}+\left(\frac{1}{G_{12}}-\frac{2 v_{12}}{E_{1}}\right) \cos ^{2} \theta \sin ^{2} \theta+\frac{\sin ^{4} \theta}{E_{2}}\right]^{-1}$

$v_{\theta}=E_{\theta}\left[\frac{v_{12}}{E_{1}}\left(\sin ^{4} \theta+\cos ^{4} \theta\right)-\left(\frac{1}{E_{1}}+\frac{1}{E_{2}}-\frac{1}{G_{12}}\right) \sin ^{2} \theta \cos ^{2} \theta\right]$

$$
\begin{aligned}
\eta_{x y, x}^{\theta}= & E_{\theta}\left[\left(\frac{2}{E_{1}}+\frac{2 \nu_{12}}{E_{1}}-\frac{1}{G_{12}}\right) \sin \theta \cos ^{3} \theta\right. \\
& \left.-\left(\frac{2}{E_{2}}+\frac{2 \nu_{12}}{E_{1}}-\frac{1}{G_{12}}\right) \sin ^{3} \theta \cos \theta\right]
\end{aligned}
$$

If the force acts in LR or LT plane, then $\theta$ in above equations represents grain angle, subscript 1 represents longitudinal direction and 2 represents radial or tangential direction. If the force acts in TR plane, then $\theta$ represents ring angle, 1 and 2 represent tangential and radial directions, respectively. 
According to the symmetry of the compliance matrixes $[S]$ and $[\bar{S}]$, five pairs of relations between Poisson's ratios and MOE in mutually orthotropic directions can be obtained as

$$
\begin{gathered}
\frac{v_{\mathrm{LR}}}{E_{\mathrm{L}}}=\frac{v_{\mathrm{RL}}}{E_{\mathrm{R}}} ; \quad \frac{v_{\mathrm{LT}}}{E_{\mathrm{L}}}=\frac{v_{\mathrm{TL}}}{E_{\mathrm{T}}} ; \quad \frac{v_{\mathrm{RT}}}{E_{\mathrm{R}}}=\frac{v_{\mathrm{TR}}}{E_{\mathrm{T}}} \\
\frac{v_{\mathrm{LR} 15}}{E_{\mathrm{LR} 15}}=\frac{v_{\mathrm{LR} 75}}{E_{\mathrm{LR} 75}} ; \quad \frac{v_{\mathrm{LR} 30}}{E_{\mathrm{LR} 30}}=\frac{v_{\mathrm{LR} 60}}{E_{\mathrm{LR} 60}}
\end{gathered}
$$

In this study, the predicted MOE, Poisson's ratios and coefficients of mutual influence by Eqs. (10)-(13) will be compared with their directly measured results to validate whether it is acceptable to treat Korean pine as ideal orthotropic material.

\section{Results and discussion}

\section{Failure patterns and stress-displacement curves}

The failure modes for compression specimens are significantly influenced by the off-axis angle. There are four typical failure modes observed in the test as shown in Fig. 3. The obtained corresponding stress-displacement curves are also given.

For specimens loaded in longitudinal direction (Fig. 3a), failure is a semi-brittle process. After a linear elastic region, a limited range of nonlinearity is observed in the curve. The maximum load carrying capacity is then reached and
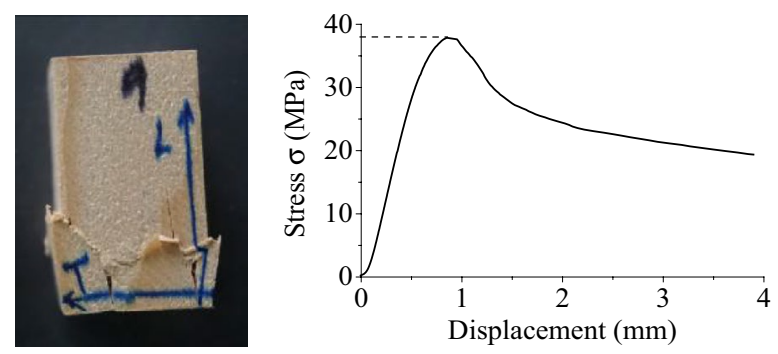

(a) Specimen in longitudinal direction
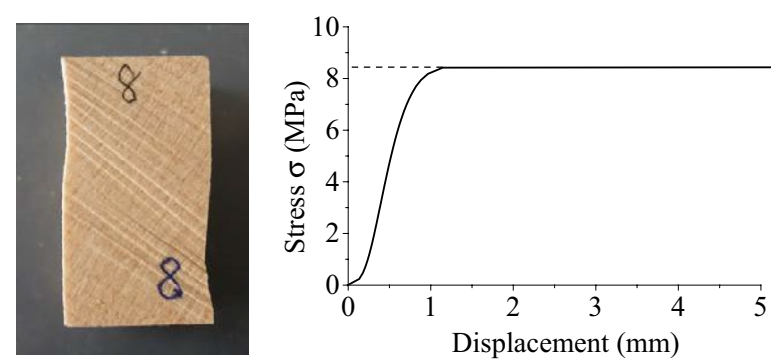

(c) Specimen with a large grain angle followed by a distinct load drop. The specimen retains some of the load resistance when the displacement continues to increase. Localized buckling and crushing of wood fibers start shortly before peak stress is reached. The final failure of the specimen was induced by the formation of axially orientation cracks. The well-known kink banding phenomenon [16] is not observed in the test probably due to the low height-to-width ratio of the tested samples.

Specimens loaded at a small grain angle (LR15 $5^{\circ}$ and LR30 ${ }^{\circ}$ ) have failure modes which are rather brittle and accompanied with a sound of breakage. Figure $3 \mathrm{~b}$ shows that the failure planes of the specimen are parallel with longitudinal fibers, approximately located at the borders from late wood of an annual ring to early wood of the next annual ring. Before slippage happened, the failure plane of the specimen is subjected to a complex stress state of shear and compression. The sudden shear failure of this plane caused the steep drop in the stress-displacement curve.

The failure mechanisms and deformation for specimens loaded at large grain angles $\left(\mathrm{LR} 45^{\circ}, \mathrm{LR} 60^{\circ}, \mathrm{LR} 75^{\circ}\right.$ and $\mathrm{LT} 45^{\circ}$ ) displayed another mode. As can be seen from Fig. 3c, the specimen distorted seriously after unloading and a distinct plastic plateau occurs in the stress-displacement curve after yielding of the material. For some wood species like spruce [1], brittle shear failure mode can be observed in $45^{\circ}$ off-axis direction even though they have similar configurations with specimens investigated here. The shear failure mode for specimens with $45^{\circ}$ off-axis angle in this study is seldom reached. In fact, the shear stresses within the specimens of $\mathrm{LR} 45^{\circ}, \mathrm{LR} 60^{\circ}, \mathrm{LR} 75^{\circ}$ and $\mathrm{LT} 45^{\circ}$
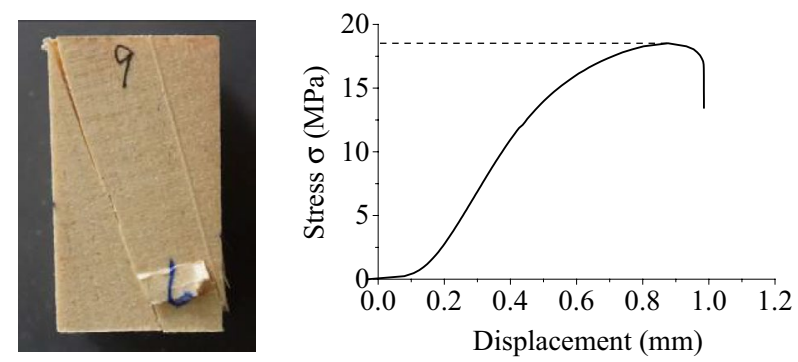

(b) Specimen with a small grain angle
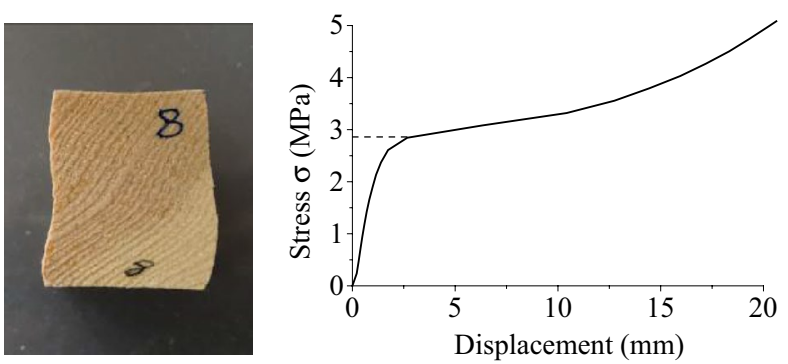

(d) Specimen in transversal direction

Fig. 3 Different failure modes of compression specimens and the obtained typical stress-displacement curves 
only causes distortion of the specimen but are not sufficient to induce shear failure.

For specimens in radial direction, tangential direction and $\mathrm{TR} 45^{\circ}$ direction, their failure patterns are alike. Because in this situation the load acts perpendicular to grain, fiber cells are collapsed and densified and the specimens can resist a higher load. The stress-displacement curve in Fig. 3d shows that after yielding of the specimen, the compressive stress hardening starts and it becomes faster with larger deformation.

In Fig. 3, the final yielding stresses for specimens with different failure modes are depicted. Specimens with first three failure modes have distinct final yielding strengths (crushing strengths) under compressive load. The exact determination of the ultimate strength for specimen in transversal direction is difficult; instead, the stress corresponding to the starting point of strain hardening stage is taken for this kind of specimen.
The failure modes for tension specimens are fairly simple. Some typical failed tension specimens are shown in Fig. 4. The failure plane for longitudinal specimen (Fig. 4a) is irregular which is caused by fiber ruptures. The hackly failure plane for specimen in $\mathrm{TR} 45^{\circ}$ direction (Fig. 4d) indicates the reduced anisotropy of TR plane. Specimens in other directions have failure planes basically parallel to the fibers' direction.

Although their rupture planes may different, tension specimens in all directions behave linearly elastic before brittle failure occurs, as examples, the stress-displacement curves for specimens loaded in $\mathrm{LR} 60^{\circ}$ and tangential directions are shown in Fig. 5.

Based on above observations, it is not difficult to summarize the following differences between tension and compression properties of Korean pine:

(a) Specimen in longitudinal direction

(b) Specimen with a small grain angle

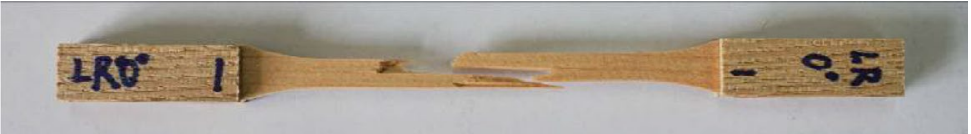

(b) Spimen with a small

(c) Specimen with a large grain angle

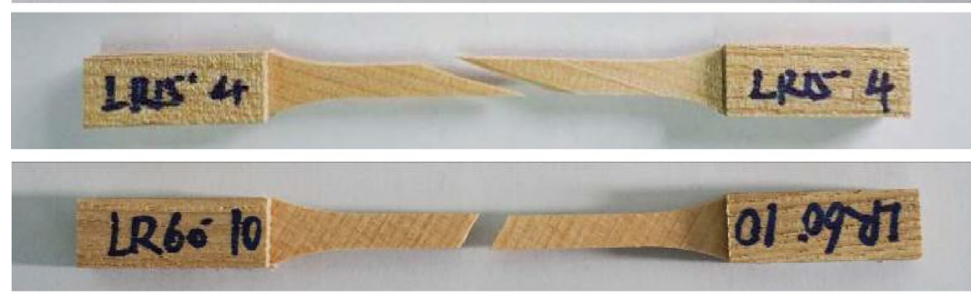

(d) Specimen in TR $45^{\circ}$ direction

Fig. 4 Typical failure modes of tension specimens loaded in different directions

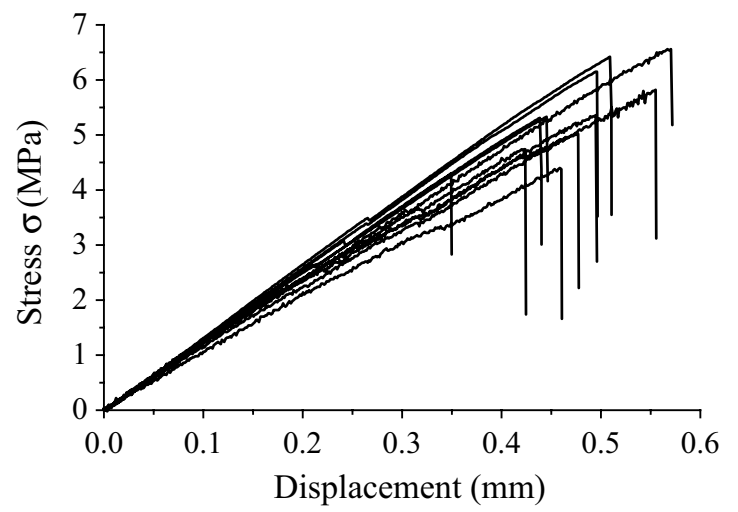

(a) Specimens in $\mathrm{LR} 60^{\circ}$ direction

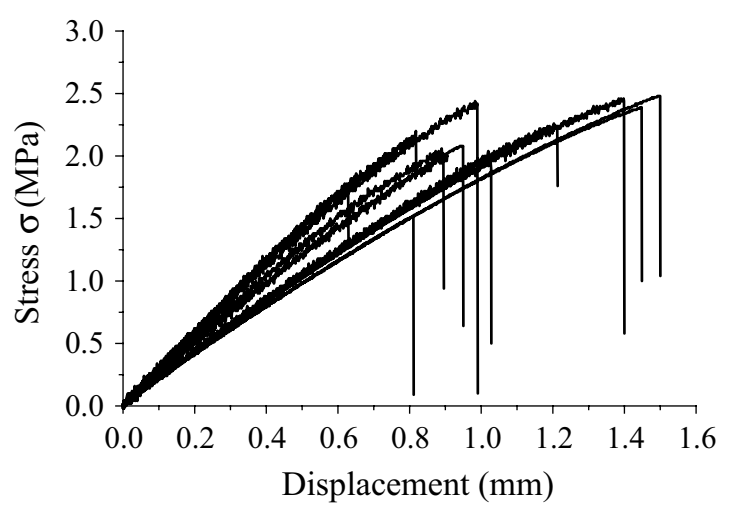

(b) Specimens in tangential direction

Fig. 5 Stress-displacement curves for tension specimens loaded in $\mathrm{LR} 60^{\circ}$ and tangential directions 


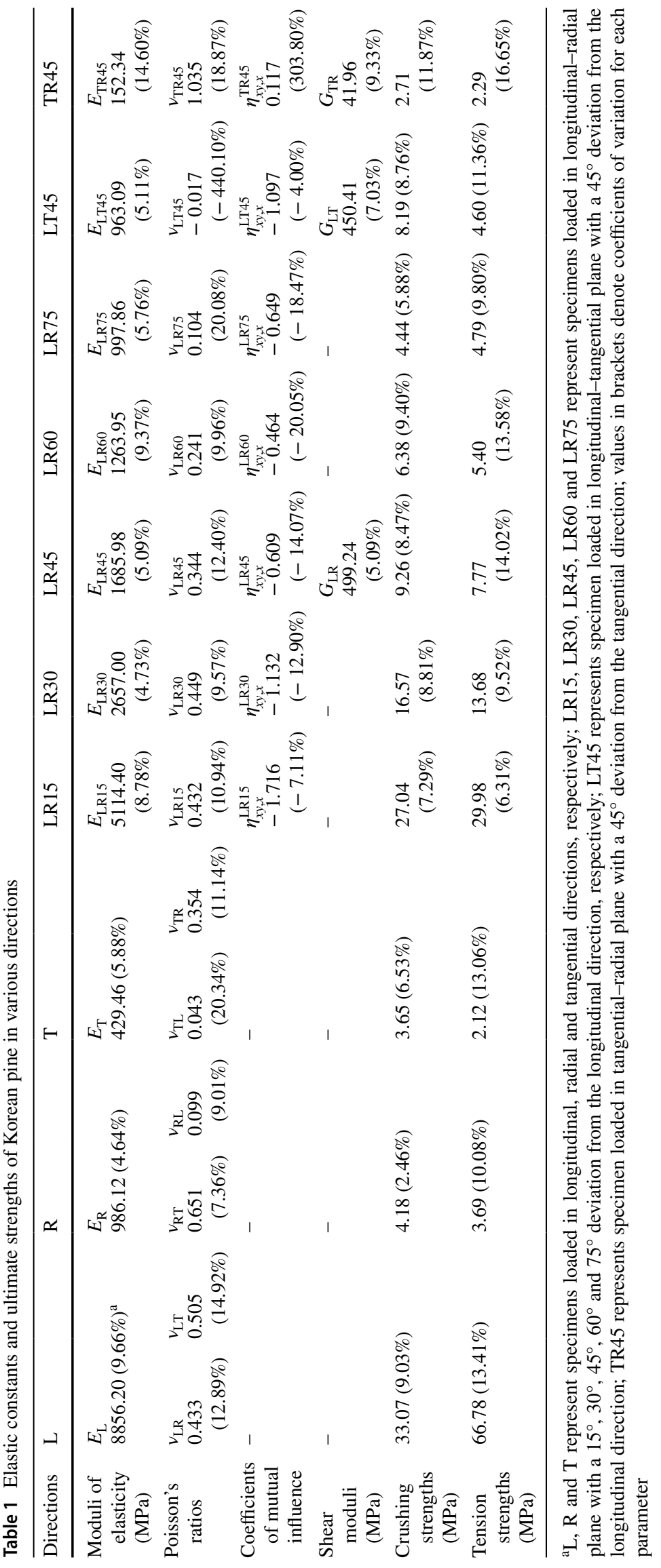


1. Specimens under tension all fail abruptly while specimens under compression have ductile failure modes if they are not loaded in small grain angles;

2. The tension specimens completely lost their continuity after failure while the compression specimens remain continuous after yielding except specimens having shear failure mode;

3. The specimens behave linearly elastic before failure by tension while there are severe nonlinearities appeared in the specimens under compression, the compressive stress-displacement curves may be softening, plastic flowing or hardening after yielding.

\section{Elastic properties}

The measured elastic constants of Korean pine including MOE, Poisson's ratios, shear moduli and coefficients of mutual influence from compression test are listed in Table 1. Both the averaged results and coefficients of variation (COV) of these parameters are given. As can be seen from Table 1, the COV for these parameters are basically below $20 \%$ which is within the common range for wooden material. The Poisson's ratio $v_{\mathrm{LT} 45}$ and coefficient of mutual influence $\eta_{x y, x}^{\mathrm{TR} 45}$ both have enormous $\mathrm{COV}$ due to their close to zero values and measurement errors.
Among the ten measured directions of Korean pine, the largest MOE is in the longitudinal direction and the lowest is in $\mathrm{TR} 45^{\circ}$ direction. MOE in longitudinal-radial plane are greater than the corresponding directions in longitudinal-tangential plane. In case of the shear moduli in three principal planes, the largest one is in LR plane $G_{\mathrm{LR}}$ which is slightly larger than that in LT plane $G_{\mathrm{LT}}$, while the shear modulus in TR plane $G_{\mathrm{TR}}$ is one order smaller. The largest Poisson's ratios measured in this study are $v_{\mathrm{TR} 45}$ which is larger than unity and the smallest one is $v_{\mathrm{LT} 45}$ which is below zero. Poisson's ratios in other directions are within the range of $0-1$. The coefficients of mutual influence for wood in three principal directions are assumed to be zero, the significant value of $\eta_{x y, x}^{\mathrm{LR} 15}$, however, indicates that even when rotated a small grain angle, the compressive stress $\sigma_{x}$ can induce considerable shear deformation [4].

The basic elastic constants of Korean pine include the MOE in three principal directions $E_{\mathrm{L}}, E_{\mathrm{R}}, E_{\mathrm{T}}$, shear moduli in three principal planes $G_{\mathrm{LR}}, G_{\mathrm{LT}}, G_{\mathrm{RT}}$ and major Poisson's ratios in three principal planes $v_{\mathrm{LR}}, v_{\mathrm{LT}}, v_{\mathrm{RT}}$. Once these parameters are derived, parameters in other directions can be theoretically obtained based on the theory of orthotropic elasticity (Eqs. 10-12). In Figs. 6, 7 and 8, the measured MOE, Poisson's ratios and coefficients of mutual influence in various off-axis directions are compared with their predicted values by Eqs. (10)-(12).

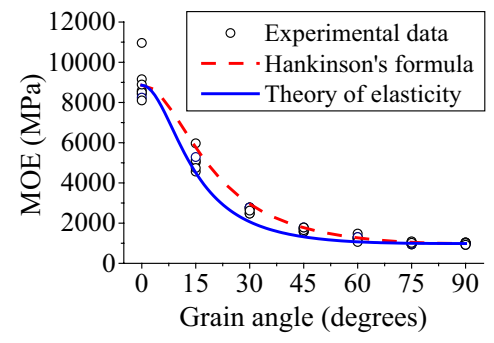

(a) LR plane

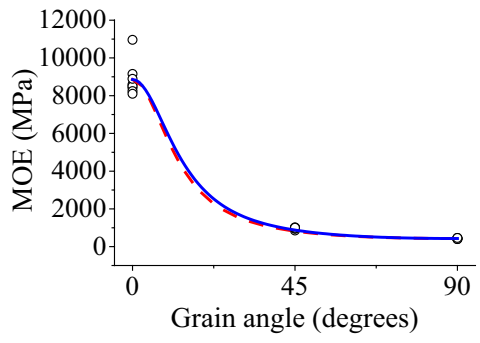

(b) LT plane

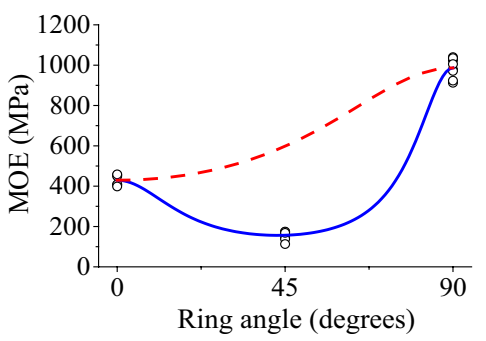

(c) TR plane

Fig. 6 Moduli of elasticity (MOE) in principal planes of Korean pine measured by compression test and the predicted results by Hankinson's formula and theory of elasticity

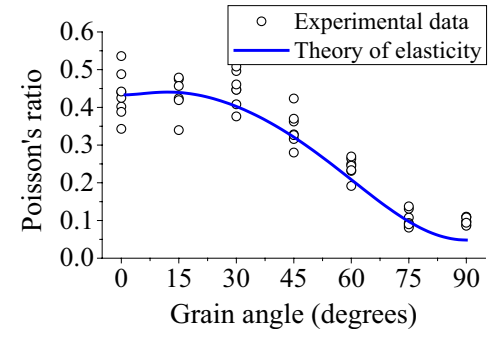

(a) LR plane

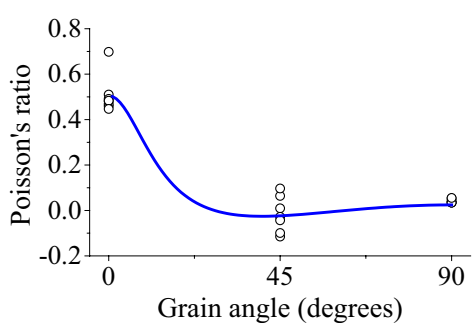

(b) LT plane

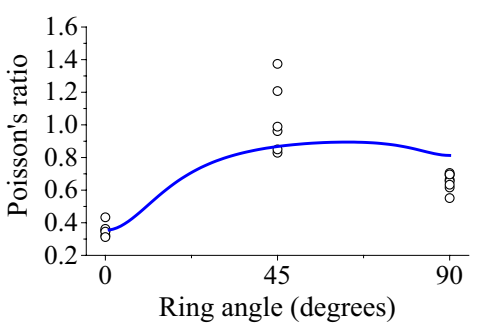

(c) TR plane

Fig. 7 Poisson's ratios in principal planes of Korean pine measured by compression test and the predicted results by theory of elasticity 


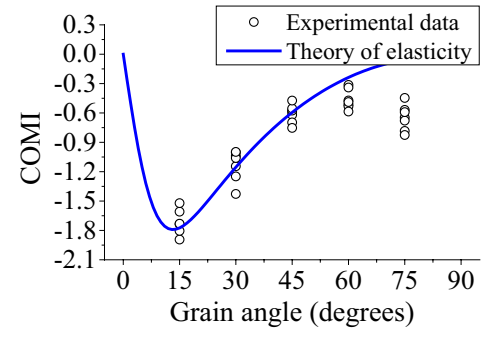

(a) LR plane

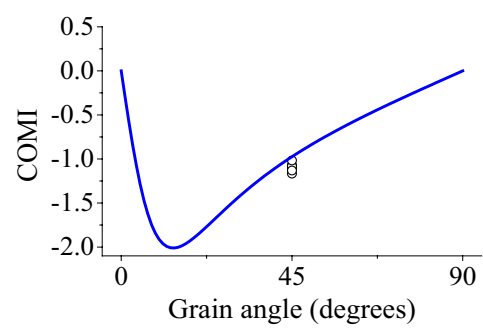

(b) LT plane

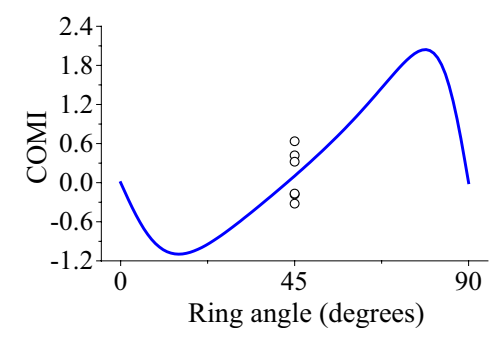

(c) TR plane

Fig. 8 Coefficients of mutual influence (COMI) in principal planes of Korean pine measured by compression test and the predicted results by theory of elasticity

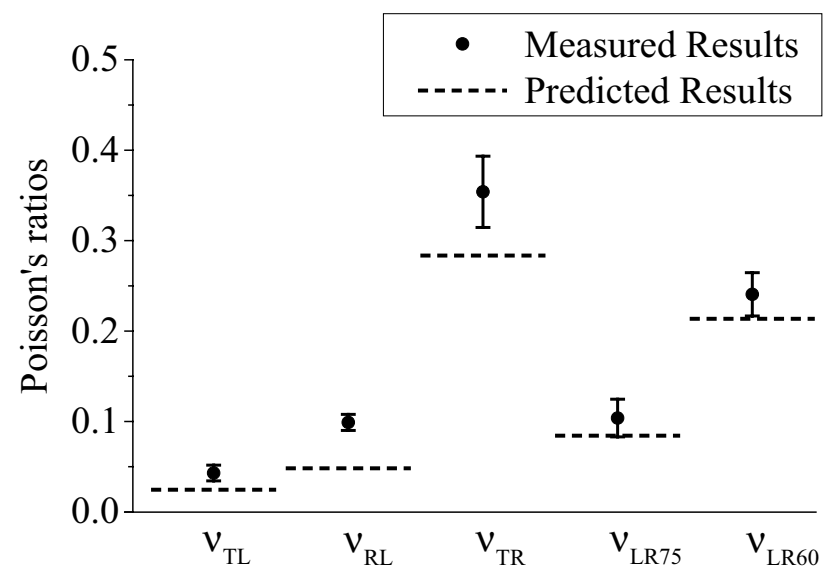

Fig. 9 Comparison of minor Poisson's ratios between measured (mean value with standard deviation) and predicted results

In Fig. 6, except from Eq. (10), the well-known Hankinson's formula as given in Eq. (14) are also drawn:

$E_{\theta}=\frac{E_{1} E_{2}}{E_{1} \sin ^{2} \theta+E_{2} \cos ^{2} \theta}$

The Hankinson's formula was initially proposed to predict the crushing strength of wood at varying angles of grain [17], Radcliffe [18] found that it can also effectively reflect the tendency of MOE changing with grain angle. As can be seen from Fig. 6a, b, both the Hankinson's formula and the formula calculated by tensor transformation can accurately reflect the tendency of MOE to decrease with increasing grain angle. Compared with transformation rule, the Hankinson's formula is preferable in practical usage because the information of shear modulus $G_{12}$ and Poisson's ratios $v_{12}$ is not needed. In TR plane, only the transformation rule can precisely capture MOE changing with ring angle, the Hankinson's formula failed to trace MOE in TR $45^{\circ}$ direction because it can only reflect a monotonic relationship of parameters changing with off-axis angle.
The dependence of Poisson's ratio on the loading direction in three principal planes is shown in Fig. 7. As can be seen, the Poisson's ratios are not monotonically changing in all principal planes. The experimentally measured dependence can be described very well by orthotropic elasticity although slight divergences are also found in radial and tangential directions. To address this discrepancy more thoroughly, Eq. (13) is examined. Five directly measured minor Poisson's ratios (averaged results with standard deviations) are compared with their predicted results calculated from the averaged measured values of the other three parameters in Eq. (13). The comparison are shown in Fig. 9. As can be seen, the measured minor Poisson's ratios are noted close to but deviate from the predicted ones. Similar deviations were described by Garab et al. [3]. This phenomenon can be attributed to the following reasons:

1. The inherent errors in the experiment and the imperfections of the material.

2. The material exhibits remarkable variation in its mechanical properties even within a small volume of the lumber from which the specimens were prepared.

3. Although wood is usually modeled as perfect orthotropic material, the curvature of annul rings makes it cylindrically orthotropic in reality [19], thus exact orthotropic cannot be guaranteed for two nominally perpendicular directions in the test.

4. The rays in radial direction contribute to the deviation of wood from orthotropic behavior.

Figure 8 compares the measured and predicted values of coefficients of mutual influence $\eta_{x y, x}$. In all principal planes, the dependence of $\eta_{x y, x}$ on off-axis angle is adequately described by the theory of orthotropic elasticity. The coefficient of mutual influence for the case of $\eta_{x y, x}^{\mathrm{LR} 75}$, however, is deviating from the predicted result. The localized deformation of the specimen during loading process, which affected the accurate measurement of shear strain $\gamma_{x y}$, was the possible reason for the observed difference. More advanced 
strain measurement techniques such as full-field measurement methods [10] should be tried in future to precisely determine the shear strain for this kind of specimen.

In this study, the congruence between experimentally measured elastic constants and formula calculated by tensor transformation is indeed high which indicates that Korean pine can be reasonably considered as orthotropic material.

\section{Ultimate strengths}

The measured ultimate strengths of Korean pine including crushing strengths and tension strengths are listed in Table 1.

The highest crushing strength and tension strength are no surprisingly resided in longitudinal direction, while the lowest crushing strength and tension strength are found in $\mathrm{TR} 45^{\circ}$ direction and tangential direction, respectively. The longitudinal-radial plane has crushing strengths and tension strengths both higher than that in the corresponding directions in longitudinal-tangential plane, probably due to the reinforcement of ray cells aligned in the radial direction [20]. The maximum coefficient of variation for both crushing strength and tension strength are with specimens in TR $45^{\circ}$ direction. This is probably due to the curvature of annual rings in this kind of specimen which increases the uncertainty of the direction in which the forces act. Although this error has been tried to avoid in preparation of specimens, yet it seems it has not been fully removed.

Comparing the crushing strength and tension strength in same directions, it is found that specimens loaded in longitudinal direction or a small grain angle such as $\mathrm{LR} 15^{\circ}$ have tension strengths higher than crushing strengths. With the increase of the grain angle, the crushing strength gradually exceeds the tension strength (except for LR75 ${ }^{\circ}$ direction). Within the ten directions studied, however, only specimens loaded in longitudinal and $\mathrm{LT} 45^{\circ}$ directions show a great difference between tension strength and crushing strength. For specimens loaded in other directions, there are only minor differences between these two properties.

The yielding strength of orthotropic materials under multi-axial stress state can be obtained from a number of yielding criteria and their applicability to wood have been extensively studied [21]. A commonly used criterion for wood was proposed by Norris [22] which was based on the Hencky-von Mises maximum distortion theory. The Norris criterion has the following form under plane stress state:

$1=\frac{\sigma_{1}^{2}}{F_{1}^{2}}-\frac{\sigma_{1} \sigma_{2}}{F_{1} F_{2}}+\frac{\sigma_{2}^{2}}{F_{2}^{2}}+\frac{\tau_{12}^{2}}{F_{12}^{2}}$

where $\sigma_{1}, \sigma_{2}$ and $\tau_{12}$ are the normal stresses and shear stress in principal directions, and $F_{1}, F_{2}$ and $F_{12}$ represent the crushing/tension strengths and shear strength in corresponding directions. For Korean pine, the crushing strengths and tension strengths in Eq. (15) can be taken from Table 1. Shear strengths in fibers' direction of Korean pine measured by Chen [23] ( $F_{\mathrm{LR}}=8.97 \mathrm{MPa}$ in LR plane and $F_{\mathrm{LT}}=9.12 \mathrm{MPa}$ in LT plane) have been adopted. The rolling shear strength $F_{\mathrm{TR}}$ for Korean pine has not been reported by researchers, thus is not discussed here.

The Hankinson's formula, as represented in Eq. (16), is also frequently used to predict the crushing strength and tension strength of wood varying with grain angle:

$F_{\theta}=\frac{F_{1} F_{2}}{F_{1} \sin ^{2} \theta+F_{2} \cos ^{2} \theta}$

In Eq. (16), $\theta$ represents the grain angle, $F_{1}$ and $F_{2}$ represent the ultimate strengths in longitudinal and transversal directions, respectively.

The measured crushing strengths and tension strengths of Korean pine in LR and LT planes together with their predicted results by Norris criterion and Hankinson's formula are shown in Fig. 10. The comparisons between the measured results and the prediction revealed that both the Norris criterion and the Hankinson's formula could effectively predict the variation of crushing strength and tension strength changing with grain angle. The crushing strength predicted by Norris criterion is more accurate than that of the Hankinson's formula, probably due to the inclusion of shear strength information in the Norris criterion. The tension strengths predicted by two equations show no visible difference. This examination is a strong validation of the used failure criterion and empirical formula.

Due to lack of rolling shear strength of Korean pine, the ultimate strengths within transverse plane are not compared with any failure criterion. Nevertheless, the measured crushing strength of Korean pine varying with ring angle in this study agrees well with the conclusions made by Ethington et al. [2] on Dahurian larch, that is, the compressive strength is highest at radial direction, lowest at intermediate angle of roughly $30^{\circ}$ to $50^{\circ}$, and middle at tangential direction. This is, however, not valid for all softwood species [3].

\section{Conclusions}

Uniaxial compression and tension tests were conducted in this study using specimens of Korean pine with various offaxis angles. The anisotropic behavior of Korean pine not only for elastic properties and ultimate strengths but also for failure mechanisms is investigated. Comparison of the measured results with those predicted from existing theoretical formulas gives the following conclusions: 


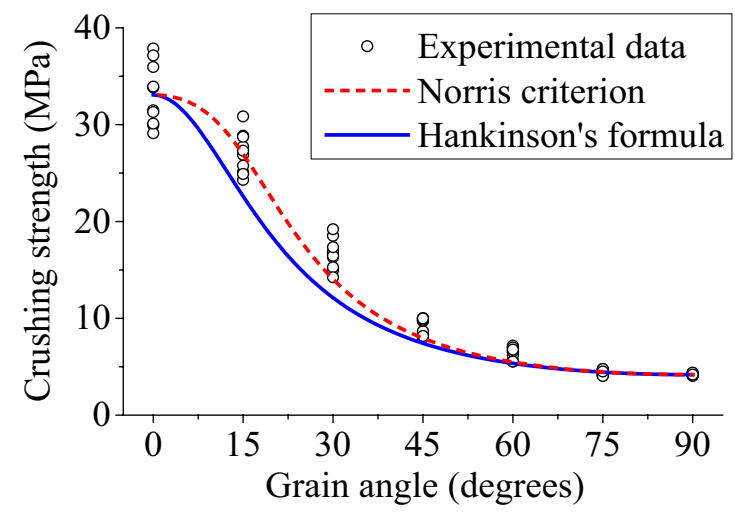

(a) Crushing strengths in LR plane

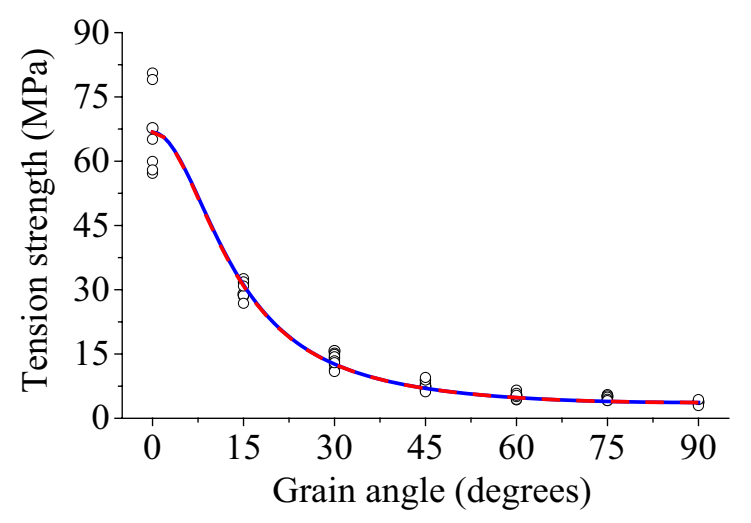

(c) Tension strengths in LR plane

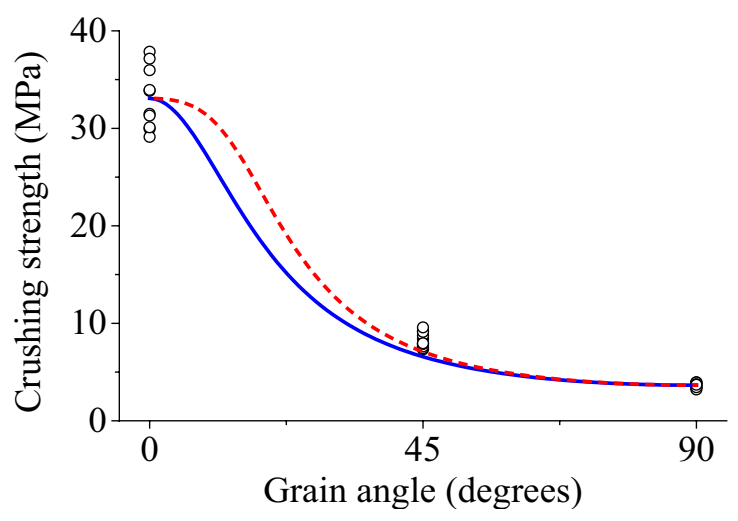

(b) Crushing strengths in LT plane

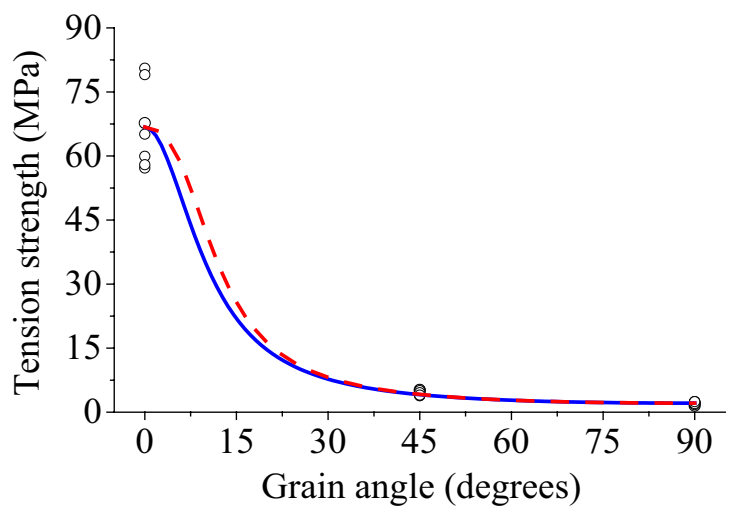

(d) Tension strengths in LT plane

Fig. 10 Crushing strengths and tension strengths of Korean pine measured in various directions in LR and LT planes and the predicted results by Hankinson's formula and Norris criterion

1. The failure modes of specimens under compressive load are highly different depending on the loading direction but can be roughly divided into two categories: specimens with small grain angles that have strain softening stage and specimens with larger grain angles that have plastic flow stage. Tension specimens all exhibit brittle failure mode.

2. Overall, the dependence of elastic properties of Korean pine including MOE, Poisson's ratios and coefficients of mutual influence on the off-axial angle can be understood well by the theory of orthotropic elasticity. The Hankinson's formula can effectively reproduce the MOE and ultimate strengths of Korean pine varying with grain angle. The Norris criterion can precisely predict the ultimate strengths in off-axis directions of Korean pine in LR and LT principal planes. These examinations are strong validations of the orthotropic assumption for Korean pine.

3. The measured parameters in this study indicated useful strength and elastic properties of Korean pine as engineering material for construction. These parameters have coefficients of variation in the common range for wood material except for those with close to zero values. The defects and cellular structure of the material need to be investigated independently in future to derive allowable strength properties for design of timber structures.

Acknowledgements The study presented in this paper was supported by National Natural Science Foundation of China for Excellent Young Scholars (NSFC 51422801), Beijing Natural Science Foundation of China (Key Program 8151003), National Natural Science Foundation of China (Key Program NSFC 51338001), National Natural Science Foundation of China (General Program NSFC 51178028) and National Key Technology R\&D Program (2015BAK01B02). The comments from Prof. S.S. Law and his help in polishing the English usage of this paper are also acknowledged.

\section{References}

1. Reiterer A, Stanzl-Tschegg SE (2001) Compressive behaviour of softwood under uniaxial loading at different orientations to the grain. Mech Mater 33:705-715 
2. Ethington RL, Eskelsen V, Gupta R (1996) Relationship between compression strength perpendicular to grain and ring orientation. Forest Prod J 46(1):84-86

3. Garab J, Keunecke D, Hering S, Szalai J, Niemz P (2010) Measurement of standard and off-axis elastic moduli and Poisson's ratios of spruce and yew wood in the transverse plane. Wood Sci Technol 44(3):451-464

4. Mascia NT, Vanalli L (2012) Evaluation of the coefficients of mutual influence of wood through off-axis compression tests. Constr Build Mater 30:522-528

5. Yoshihara H, Ohta M (2000) Estimation of the shear strength of wood by uniaxial-tension tests of off-axis specimens. J Wood Sci 46:159-163

6. Zhang W, Sliker A (1991) Measuring shear moduli in wood with small tension and compression samples. Wood Fiber Sci 23:58-68

7. Nadir Y, Nagarajan P, Midhun AJ (2014) Measuring elastic constants of Hevea brasiliensis using compression and Iosipescu shear test. Eur J Wood Wood Prod 72:749-758

8. Wang L, Lu Z (2004) Twelve elastic constants of Betula platyphylla Suk. Forest Stud China 6:37-41

9. Janowiak JJ, Pellerin RF (1992) Shear moduli determination using torsional stiffness measurements. Wood Fiber Sci 24(4):392-400

10. Grédiac M (2004) The use of full-field measurement methods in composite material characterization: interest and limitations. Compos Part A Appl S 35:751-761

11. Xavier J, Avril S, Pierron F, Morais J (2007) Novel experimental approach for longitudinal-radial stiffness characterisation of clear wood by a single test. Holzforschung 61:573-581

12. Xavier J, Avril S, Pierron F, Morais J (2009) Variation of transverse and shear stiffness properties of wood in a tree. Compos Part A Appl S 40:1953-1960
13. ASTM D 143-09 (2009) Standard test methods for small clear specimens of timber. American Society for Testing and Materials, West Conshohocken, pp 2-3

14. Yoshihara H (2009) Prediction of the off-axis stress-strain relation of wood under compression loading. Eur J Wood Wood Prod 67(2): 183-188

15. Jones RM (1975) Mechanics of composite materials. Taylor \& Francis, Philadelphia, pp 37-40

16. Benabou L, Sun Z (2015) Numerical study of anisotropic failure in wood under large deformation. Mater Struct 48:1977-1993

17. Hankinson RL (1921) Investigation of crushing strength of spruce at varying angles of grain. Air Serv Inf Circ 259:1-15

18. Radcliffe BM (1965) A theoretical evaluation of Hankinson's formula for modulus of elasticity of wood at an angle to the grain. Q Bull Michigan Agr Exp Sta 48:286-295

19. Aicher S, Dill-Langer G, HöFflin L (2001) Effect of polar anisotropy of wood loaded perpendicular to grain. J Mater Civil Eng 13(1):2-9

20. Holmberg S, Persson K, Petersson H (1999) Nonlinear mechanical behaviour and analysis of wood and fibre materials. Comput Struct 72:459-480

21. Cabrero JM, Blanco C, Gebremedhin KG, Martin-Meizoso A (2012) Assessment of phenomenological failure criteria for wood. Eur J Wood Wood Prod 70:871-882

22. Norris CB (1962) Strength of orthotropic materials subjected to combined stresses, Report No. 1816. Forest Products Laboratory, Madison

23. Chen Z (2011) Behaviour of typical joints and the structure of Yingxian Wood Pagoda (in Chinese). Dissertation, Harbin Institutive of Technology, China 